Utah State University

DigitalCommons@USU

$12-1-1990$

\title{
Crit II Electric, Magnetic, and Density Measurements Within an lonizing Neutral Stream
}

\author{
C. M. Swenson
}

M. C. Kelley

F. Primdahl

K. D. Baker

Follow this and additional works at: https://digitalcommons.usu.edu/sdl_pubs

\section{Recommended Citation}

Swenson, C. M.; Kelley, M. C.; Primdahl, F.; and Baker, K. D., "Crit II Electric, Magnetic, and Density Measurements Within an lonizing Neutral Stream" (1990). Space Dynamics Lab Publications. Paper 125. https://digitalcommons.usu.edu/sdl_pubs/125

This Article is brought to you for free and open access by the Space Dynamics Lab at DigitalCommons@USU. It has been accepted for inclusion in Space Dynamics Lab Publications by an authorized administrator of DigitalCommons@USU. For more information, please contact digitalcommons@usu.edu.

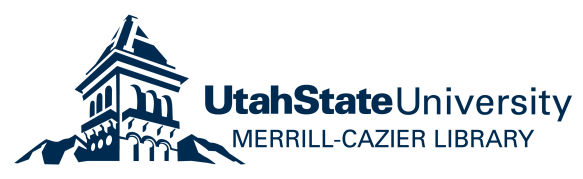




\section{CRIT II ELECTRIC, MAGNETIC, AND DENSITY MEASUREMENTS WITHIN AN IONIZING}

Swenson, C. M., M. C. Kelley, F. Primdahl, and K. D. Baker. 1990. "Crit II Electric, Magnetic, and Density Measurements within an Ionizing Neutral Stream." Geophysical Research Letters 17 (12): 2337 - 40. doi:10.1029/GL017i013p02337.
NEUTRAL STREAM

C. M. Swenson and M. C. Kelley

Cornell University

F. Primdahl

Danish Space Research Institute

K. D. Baker

Utah State University
Abstract. Measurements from rocket borne sensors inside a high velocity neutral barium beam show a factor of six increase in plasma density in a moving ionizing front. This region was co-located with intense fluctuating electric fields $(\delta \mathrm{E} \approx 300 \mathrm{mV} / \mathrm{m})$ at frequencies well under the lower hybrid frequency for a barium plasma. Large quasi-DC electric and magnetic field fluctuations were also detected with a large component of the current and the electric field parallel to $\mathbf{B}_{0}$. An Alfvén wave with a finite electric field component parallel to the geomagnetic field was observed to propagate along $\mathbf{B}_{\mathbf{o}}$, where it was detected by an instrumented sub-payload.

\section{Introduction}

The critical ionization velocity effect (CIV) can occur when a streaming neutral gas penetrates a magnetized plasma at high velocity. It is a process by which the kinetic energy of the neutral stream is tapped to provide energy for ionizing itself. This effect was first proposed by Alfvén [Alfvén, 1954]. The magnetized plasma acts as a catalyst for the energy transfer process and also absorbs the excess momentum of the reacting neutrals. Recent reviews of both laboratory and space based CIV experiments have been presented in the literature [Newell, 1985; Torbert, 1990]. The consensus is that energy of the streaming neutrals must be transferred into heated electrons, which then become efficient at ionizing the neutrals of the stream.

\section{Experiment}

The CRIT II sounding rocket was launched on May 4, 1989 at 1:24:10 UT from Wallops Island, Virginia. Two shaped charge barium releases were made about $90 \mathrm{sec}-$ onds apart at $423.5 \mathrm{~km}$ and $407.5 \mathrm{~km}$, in darkness to eliminate photo ionization as a contributing process, and both at a $\approx 45^{\circ}$ angle to the magnetic field. The main payload was positioned within the path of the neutrals to directly observe the processes within the stream, while the subpayload was positioned outside of the stream along the connecting geomagnetic field (Figure 1). Torbert [Torbert et al., 1990] presents a more detailed description of the experiment geometry along with in situ electron and ion par-

lopyright 1990 by the American Geophysical Union. ticle energy measurements. The experiment also included ground-based optical observations [Stenbaek-Nielsen et al., 1990] as well as the in situ electric, magnetic, and plasma density measurements reported here.

The electric field probe on the main payload consisted of three sets of extendable dipole antennas from which eleven independent measurements were made. A calibrated fluxgate magnetometer was used to obtain perturbation measurements due to the ionizing neutral beam. The absolute plasma density was determined from plasma frequency probe measurements of the upper-hybrid frequency. The sub-payload was about $1.76 \mathrm{~km}$ from the region of interaction around the main payload during the first burst and made particle flux, electric, and magnetic field measurements. Sub-payload data will not be shown due to page limitations.

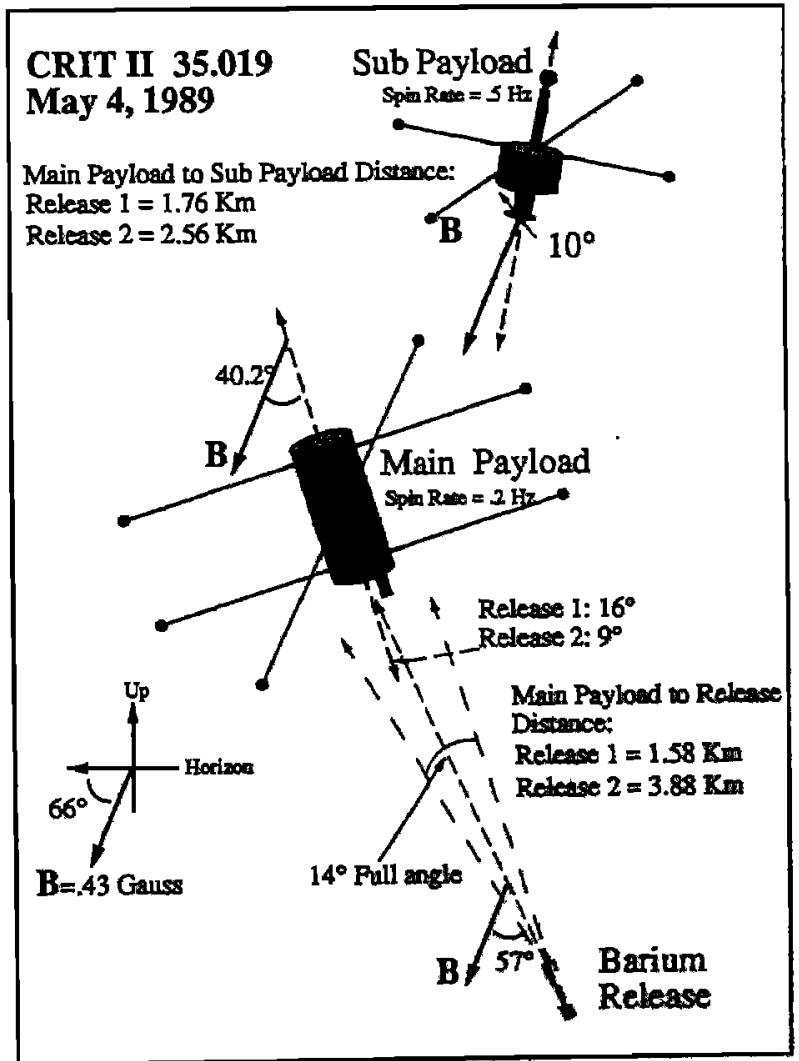

Fig. 1. Diagram of the experiment configuration. 


\section{Data Presentation}

Data from the main payload are presented in Figure 2. The time axis is referenced to the beginning of the burst data storage mode of the telemetry system or 348.262 seconds after launch. A velocity distribution can be inferred for the neutral barium stream from similar releases of strontium [Torbert, 1990]. The bulk of the neutral barium is calculated to arrive at the main payload at 0.132 s. Plasma density is plotted in the first panel of Figure 2. The plasma density is seen to increase from a background density of $5.4 \times 10^{5} \mathrm{~cm}^{-3}$ to a peak of about $2.8 \times 10^{6} \mathrm{~cm}^{-3}$ and drop to $1.1 \times 10^{4} \mathrm{~cm}^{-3}$, which is below the original background level. The initial disturbances in plasma density $(0.112 \mathrm{~s})$ correspond to the arrival of $16 \mathrm{~km} / \mathrm{s}$ neutral barium. This is fastest than the $13.5 \mathrm{~km} / \mathrm{s}$ cut-off predicted by the strontium based velocity distribution. It is therefore uncertain if collisionally accelerated neutral oxygen, unusually fast neutral barium, or both are interacting at those times. For reference Alfvén's critical velocity for barium, $V_{c}$, is $2.8 \mathrm{~km} / \mathrm{s}$. The end of the region of increased density, around $0.175 \mathrm{~s}$, corresponds to a velocity of $9.7 \mathrm{~km} / \mathrm{s}$. The time period from 0.112 to 0.175 seconds defines a region we
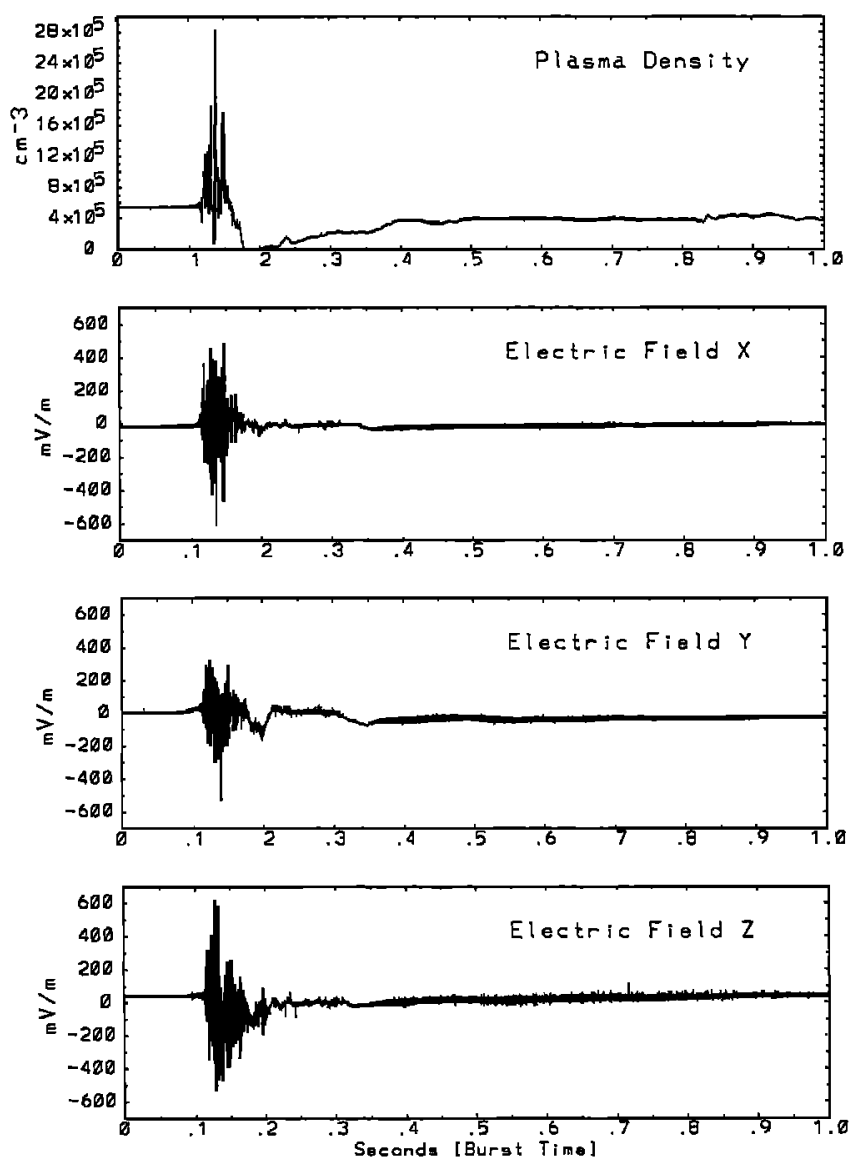

Fig. 2. Measurements at the main payload of plasma density (0-6 kHz Nyquist, panel 1) and the electric field in three orthogonal directions $(0-12 \mathrm{kHz}$ Nyquist, panels 24). call the ionization front since the largest density increases and field fluctuations occur between these times. There is every reason to believe that this active region rides with the neutral barium, implying a 768 meter spatial width along the stream at $0.135 \mathrm{~s}$. For comparison, the gyro radius of barium ions created at this time is $\approx 360 \mathrm{~m}$. The rise time of the spiky features $(\approx 1 \mathrm{~ms})$ are well within the bandwidth of the plasma frequency probe and must be considered as a real physical effect. These fluctuations could represent spatial regions of intense ionization within the front. The required production rate can be estimated from the density increase and the time since the arrival of the front. This gives a peak production rate of $8 \times 10^{7} \mathrm{~cm}^{-3} \mathrm{~s}^{-1}$ within these spikes which is larger than, but consistent with the lower limit inferred from electron measurements $\left(\gtrsim 10^{6} \mathrm{~cm}^{-3} \mathrm{~s}^{-1}\right.$ [Torbert et al., 1990]). Even this lower limit is greater than what would be expected from collisions, or stripping, with the neutral atmosphere [Stenbaek-Nielsen et al., 1990]. The depletion region, after $0.18 \mathrm{~s}$, occurs well after the passage of the ionization front. The formation of this 'hole' in the ionosphere may be due to the heavy barium ions forcing out the lighter ionospheric oxygen ions. As the barium ions leave the region a depleted plasma 'hole' is left behind which slowly recovers on the ion acoustic time scale. This phenomena has been shown to occur in computer simulations of chemical releases [Schunk and Szuszczewicz, 1990].

Electric field data are presented in panels 2 through 4 of Figure 2. Due to an attitude control failure, the spin axis of the main payload was 15.8 degrees off alignment with the neutral stream. We will consider this misalignment of the measurement coordinates small and ignore it for this qualitative discussion. Panels 2 and 3 contain electric field measurements made nearly perpendicular to the neutral stream while, panel 4 is the component of the electric field parallel to the stream. The perpendicular axes are rotated approximately 40 degrees from the $V_{B_{a}} \times B_{0}$ 。 direction where $\mathbf{V}_{\mathrm{Ba}}$ is the velocity vector of the barium stream and $\mathbf{B}_{\mathrm{o}}$ is the geomagnetic field vector. First, consider the most general wave activity in these plots. During the initial growth period from 0.11 to $0.12 \mathrm{~s}$, (see Figure 3 for better time resolution) the waves are narrow banded $(\delta f / f \approx 0.1)$ and grow with about a $0.3 \mathrm{~ms}$ time constant $\left(\gamma \approx 0.1 \omega_{0}\right.$ where $\omega_{0}$ is the observed average wave frequency and $\gamma$ is the growth rate). The wave frequency dropped from $1.6 \mathrm{kHz}$ to $\approx 300 \mathrm{~Hz}$. The wave amplitude peaked at $\approx 400 \mathrm{mV} / \mathrm{m}$, an amplitude at which the process apparently saturated ( $0.12 \mathrm{~s})$. The 'saturation' wave pattern changed character at the time the most intense electron flux was observed and the bulk of the neutrals arrived [Torbert et al., 1990]. The electric field after this time had lower amplitudes and less coherent structure.

The quasi-DC electric field associated with the ionization front is somewhat difficult to detect in Figure 2 because of the wave activity. After filtering the data, we find peak quasi-DC electric fields across the neutral stream of $\approx 50$ $\mathrm{mV} / \mathrm{m}$ and $\approx 170 \mathrm{mV} / \mathrm{m}$ parallel to the stream. The waves of the ionization front seem to be associated with the gradient of this parallel field and the arrival of the bulk of the barium with its peak value. Transformation of these quasi-DC measurements to a geomagnetically aligned coordinate system shows that a $100 \mathrm{mV} / \mathrm{m}$ (peak value) magnetically parallel electric field was present within the front. 
A neutral beam aligned field, with a large geomagnetically aligned component, was also detected in the CRIT I experiment [Brenning et al., 1990a]. In that experiment the ratio of the quasi-DC field to the wave fluctuations was $\approx 3$ where in this case the ratio was $\approx 0.3$. This observation may be related to the fact that the CRIT II experiment was much more efficient at ionizing barium than CRIT I [Stenbaek-Nielsen et al., 1990].

Magnetic field deviations associated with the release from the ambient geomagnetic field are shown in panels 1 through 4 of Figure 3. Panels 1 and 2 show perturbation measurements of the magnetic field across the neutral stream, with panel 2 in the $\mathbf{V}_{\mathrm{Ba}_{\mathbf{a}}} \times \mathbf{B}_{\text {o direction. Panel }}$ 3 presents the parallel fluctuations and panel 4 the total magnetic field magnitude. With the passage of the ionization front, large $(\approx \mathbf{3 0 0} \mathrm{nT})$ deviations were observed in each of the directions perpendicular to the neutral stream while virtually no fluctuations were detected in the parallel direction.

At 0.135 seconds the magnetic perturbation rapidly changed direction from negative to positive indicating the presence of a strong current (panel 2). If we assume the observed magnetic perturbations result from a current system that is stationary in the frame of the barium stream and cross stream derivatives of $B_{z}$ are small, then an esti-
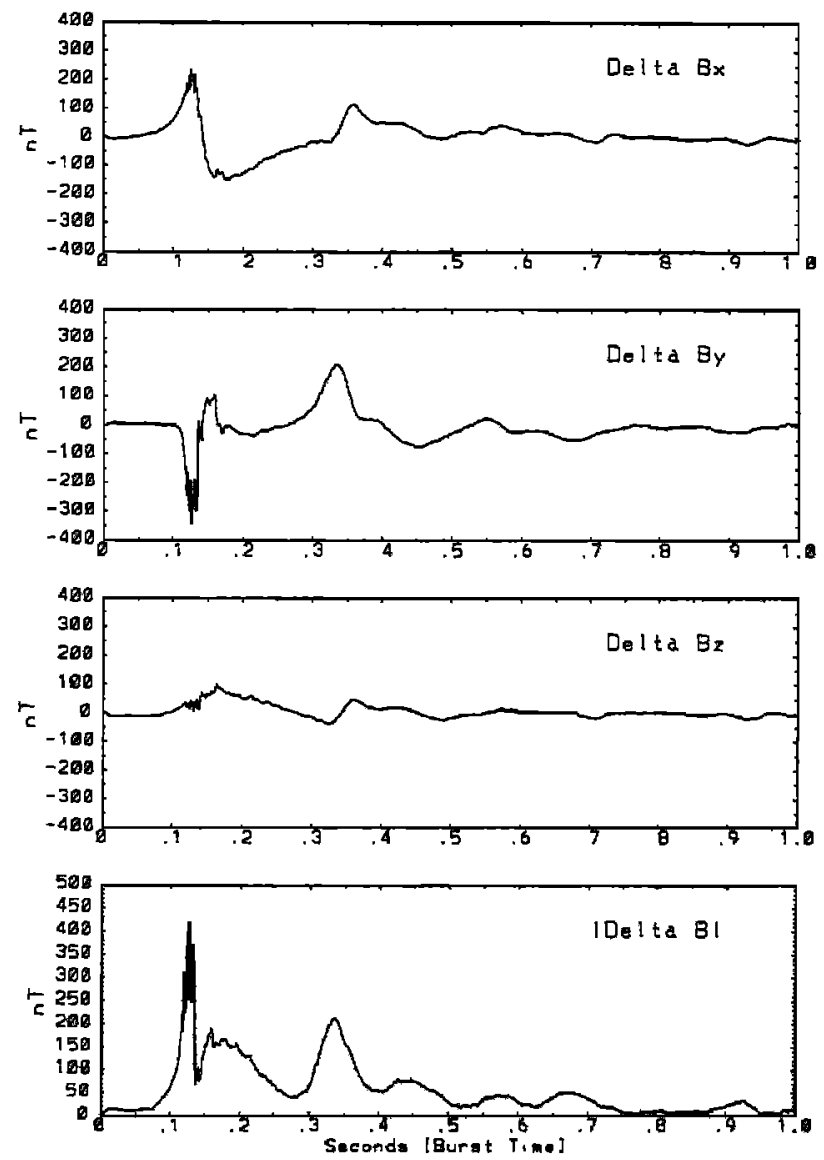

Fig. 3. Measurements at the main payload of perturbations in the magnetic field in three orthogonal directions (0-600 $\mathrm{Hz}$ Nyquist, panels 1-3) and the total magnitude of the perturbations (0-600 Hz Nyquist, panel 4). mate of two of the components of the curl of $\mathbf{B}$ can be made using $\frac{\partial \mathrm{B}_{\mathrm{x}}}{\partial z}$ and $\frac{\partial \mathrm{B}_{\mathrm{y}}}{\partial z}$, and the equation $\frac{1}{\mathrm{~V}_{\mathrm{B}}} \frac{\partial \mathrm{B}_{1}}{\partial t}$. Using these measurements and Ampere's law we can only estimate the currents perpendicular to $V_{B \Delta}$ and can say nothing about the parallel currents. This calculation gives $9.5 \mathrm{~mA} / \mathrm{m}^{2}$ at $0.135 \mathrm{~s}$. This current lies mostly in the plane of $\mathbf{B}_{\mathbf{e}}$ and $V_{B_{2}}$ and could be completely aligned with the magnetic field since the parallel component of the curl of $\mathbf{B}$ is not observable under these assumptions. The field aligned current that would be consistent with the observations is $11 \mathrm{~mA} / \mathrm{m}^{2}$. This current density is $10^{3}$ times larger than thase typically measured in auroral arcs. Currents in the $\mathbf{V}_{\mathrm{Ba}} \times \mathbf{B}_{0}$ direction which could exchange momentum in the axial direction of the beam would show up as derivatives in panel 1 and are clearly weaker than those in panel 2. The smaller perturbations riding on the first pulse seen in panel 2 indicate an oscillating current system within the ionization front.

In addition to an energy balance, there must also be a balance of momentum in the CIV process. The momentum of the reacting neutrals is thought to be transported to the surrounding ionosphere via Alfvén waves [Haerendel, 1982]. The sub-payload experiment was designed to detect this type of phenomena. We found the ratio of the perpendicular electric and perpendicular magnetic field fluctuations at the sub-payload in MKS units to be $\approx 276 \mathrm{~km} / \mathrm{s}$ at the same time the ionization front passed the main payload. This is close to the local Alfvén speed of $300 \mathrm{~km} / \mathrm{s}$. One of the more intriguing observations at both the main and sub-payloads is that two and possibly three Alfven wave packets were observed after the passage of the ionization front. These Alfvén wave pulses were separated in time by barium cyclotion periods $\left(2 \pi / \Omega_{\mathrm{Bz}^{+}}\right)$. Magnetic signatures of these pulses at the main payload are most easily observed in panel 4 of Figure 3. The ionization front, mowing with the barium neutrals, mould have moved out of contact with the field lines near the main payload. This means that pulses following the initial ionization front are associated with magnetized barium ions, created within the front, returning after their first and second gyro orbits. These electric and magnetic field observations after the passage of the ionization front are in agreement with the gyrating barium swarm theary developed by Brenning [Brenning et al., 1990b].

Figure 4 shows the porwer spectrum and the associated time series of the electric field parallel to $V_{B a}$ during the passage of the ionization front. The strongest fluctuations observed during the passage of the front are in a narrow band around $300 \mathrm{~Hz}$. For reference the cyclotran frequencies of oxygen, hydrogen, helium, and the lower hybrid frequencies for a barium plasma $(2362 \mathrm{~Hz})$ and an oxyger plasma ( $6914 \mathrm{~Hz}$ ) have been noted on the same figure. The frequency of these waves cannot be associated with either of these lower hybrid frequencies. The lower hybrid waves traditionally had boen thought of as the mechanism for heating the electrons in the CIV. We have, without success, endeavored to bypothesize a wave phase velocity consiatent with a lower hybrid mode which would have a Doppler shifted frequency oqual to the observed value. Thes measurements thus indicate that ather inotabilities mast be considered. The abeence of a clows control of the physics by lower hybrid turbulence is in agreement with other in 

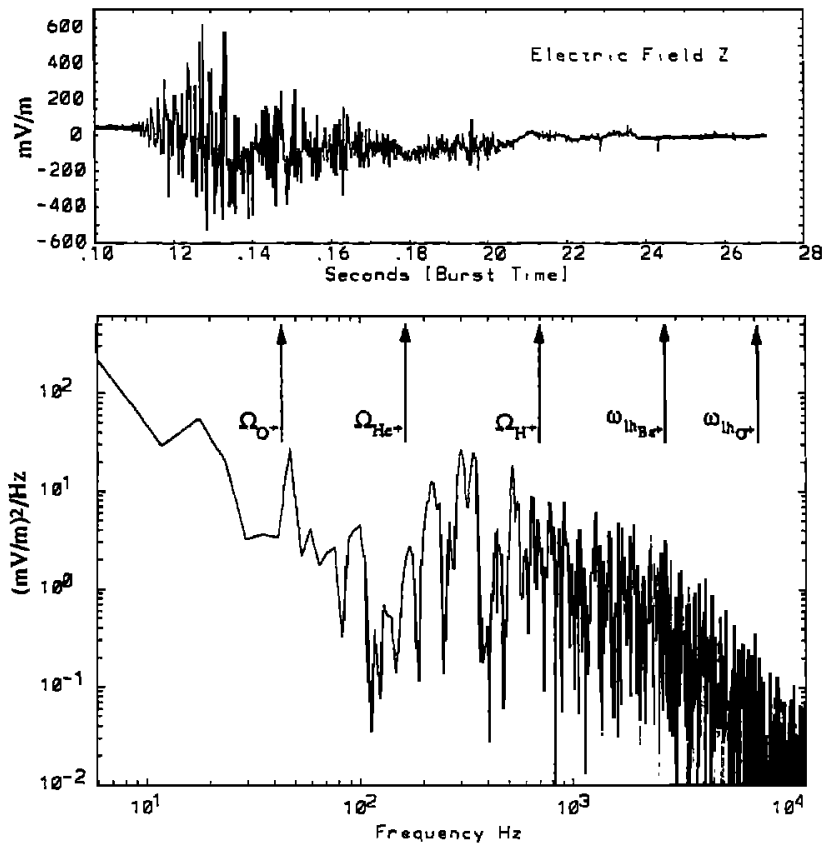

Fig. 4. Electric field measurement parallel to the neutral barium stream during the passage of the ionization front (0-12 kHz Nyquist, panel 1) and the corresponding power spectra (panel 2) of the time series.

situ CIV observations which also found intense signals well below the lower hybrid range [Kelley et al., 1986].

\section{Conclusion}

The initjal analysis of the CRIT II plasma density, electric, and magnetic field measurements indicate that significant plasma density increases were measured within the neutral stream corresponding with the arrival of the fastest neutral barium. This region is thought to be the ionization front of a CIV process. Strong, low frequency $(300 \mathrm{~Hz})$ electric and magnetic wave activity accompanied these plasma density enhancements. The region of intense waves and anomalous ionization was limited to a region corresponding to the neutral velocity in the range 16 $9.7 \mathrm{~km} / \mathrm{s}$. This is coincident with the superfast part of the beam and possibly accelerated atmospheric particles. At these velocities ionization by stripping against neutral oxygen is energetically possible albeit at a low rate and may imply that the CIV process needs this as a seeding mechanism. This is one possible explanation for the high variability of CIV experiments in space, since the neutral oxygen density varies by two orders of magnitude at the altitudes of the experiments. This velocity range is well above the the so-called critical velocity threshold for bar$\operatorname{ium}(2.8 \mathrm{~km} / \mathrm{s})$. The strongest wave activity is not associated with the lower hybrid frequency as predicted by theory but is concentrated in a frequency band from 200 to 600 $\mathrm{Hz}$. A current density of $9.5 \mathrm{~mA} / \mathrm{m}^{2}$ across the neutral stream can be inferred from the magnetometer measurements. A significant quasi-DC electric field parallel to the geomagnetic field was also detected within the front. The sub-payload positioned outside of the path of the ionization front, but in contact with it along the geomagnetic field, detected large electric and magnetic fluctuations consistent with Alfvénic pulses. The time spacing of these pulses was equal to the barium cyclotron period. Similar fluctuations were observed at the main payload and are thought to be due to a bunching of magnetized barium ions which gyrate though the background ionospheric plasma.

Acknowledgments. The authors are grateful to Robert Green of Cornell University, Mark Jensen and Jay Ballard of Utah State University, and Lars Bylander and Christer Beckmann of the Royal Institute of Technology for their engineering of the various instruments. We also thank $D_{r}$. Nils Brenning for his contributions and insight. The Cornell research was funded under NASA grant NSG-6020.

\section{References}

Alfvén, H., On the Origin of the Solar System, Oxford University Press, New York, 1954.

Brenning, N., C. Falthammar, G. Haerendel, M. Kelley, G. Marklund, R. Pfaff, J. Providakes, H. Stenbaek-Nielsen, C. Swenson, R. Torbert, and E. Wescott, Interpretation of the electric fields measured in an ionospheric critical ionization velocity experiment., $J$. Geophys. Res., in press, 1990 a.

Brenning, N., J. Providakes, M. Kelley, and C. Swenson, Barium swarm: An ionospheric ac generator in CRIT I, J. Geophys. Res., in press, $1990 b$.

Haerendel, G., Alfvén's critical velocity effect tested in space, Z. Naturforsch, 37a, 728-735, 1982.

Kelley, M., R. Pfaff, and G. Haerendel, Electric field measurements during the condor critical velocity experiment, J. Geophys. Res., 91, 9939-9946, 1986.

Newell, P., Review of the critical ionization velocity effect in space, Rev. Geophys., 23, 93-104, 1985.

Schunk, R. W. and E. P. Szuszczewicz, Plasma expansion characteristics of ionized clouds in the ionosphere: macroscopic formulation, J. Geophys. Res., in press, 1990.

Stenbaek-Nielsen, H., E. Wescott, G. Haerendel, and A. Valenzuela, Optical observations on the CRIT-II critical ionization velocity experiment, Geophys. Res. Lett., 17(10), 1601-1604, 1990.

Torbert, R., Review of critical velocity experiments in the ionosphere, Adv. Space Res., 10(7), 747-758, 1990.

Torbert, R., C. Kletzing, K. Liou, and D. Rau, Particle observations in a critical velocity experiment, Geophys. Res. Lett., in press, 1990.

K. D. Baker, Utah State University, Logan, Utah 84322 M. C. Kelley and C. M. Swenson, School of Electrical Engineering, Cornell University, Ithaca, New York 14853

F. Primdahl, Danish Space Research Institute, DK-2800 Lyngby, Denmark

(Received June 18, 1990;

Revised August 22, 1990;

Accepted August 22, 1990) 